\section{TET2 promoter DNA methylation and expression analysis in pediatric B-cell acute lymphoblastic leukemia}

\author{
Ewa Musialik, ${ }^{1}$ Mateusz Bujko, ${ }^{1}$ \\ Agnieszka Wypych, ${ }^{2}$ Michał Matysiak, \\ Janusz Aleksander Siedlecki ${ }^{1}$ \\ 1Department of Molecular and \\ Translational Oncology, Maria \\ Sklodowska-Curie Memorial Cancer \\ Center and Institute of Oncology, \\ Warsaw; ${ }^{2}$ Department of Pediatric \\ Haematology and Oncology, Medical \\ University of Warsaw, Poland
}

\section{Abstract}

TET2 is a novel tumor suppressor gene involved in several hematological malignancies of myeloid and lymphoid origin. Besides loss-of-function mutations and deletions, hypermethylation of the $\mathrm{CpG}$ island at the TET2 promoter was found in human cancer. Previous analysis revealed no TET2 mutations in acute lymphoblastic leukemia (ALL). Since the TET2 promoter methylation status in pediatric ALL has not been reported, the aim of the present study was to determine if promoter hypermethylation may be a mechanism of TET2 inactivation in a group of pediatric ALL cases. Methylation of TET2 promoter region in one (1/45) ALL B-common patient was detected by methylation specific polymerase chain reaction (PCR) and subsequently analyzed by bisulfite sequencing. We found no correlation between promoter methylation and gene expression, measured by quantitative reverse transcriptase-PCR, however the level of TET2 expression in ALL group was significantly decreased compared to children's normal peripheral blood mononuclear cells and isolated B-cells. TET2 promoter hypermethylation seems to have limited clinical relevance in childhood B-cell ALL due to its low frequency.

\section{Introduction}

B-cell acute lymphoblastic leukemia (ALL) is the most common type of cancer in children. The hallmarks of leukemia are chromosomal translocations, however other genetic lesions play a role in pathogenesis as well. Recently some new important genetic aberrations have been found in hematological neoplasms including TET2 mutations.

TET2 is a member of Ten-Eleven-
Translocation gene family that encodes 2-oxoglutarate-dependent and iron-dependent dioxygenase. It catalyzes the conversion of 5-methylcytosine to 5-hydroxymethylcytosine in DNA and is involved in the DNA methylation regulation machinery and hematopoiesis., ${ }^{1,2}$ Reduction in TET2 expression triggers increase in number of hematopoietic stem cells (HSCs), enhances their self-renewal capacity in vitro and confers competitive advantage over wildtype HSCs in vivo. The altered differentiation of hematopoietic cells is predominantly skewed toward myeloid lineage, however inactivation of TET2 in mouse models affects both myeloid and lymphoid differentiation. ${ }^{3}$

TET2 was identified as a tumor suppressor gene and its mutations were found in adult patients with myelodysplastic syndrome (MDS), myeloproliferative neoplasms (MPN), acute myeloid leukemia (AML) and chronic myelomonocytic leukemia (CMML) with particularly high frequency (up to 65\%) in patients with CMML. ${ }^{4-15}$ Some clinical reports showed prognostic value of TET2 mutations in these malignancies. ${ }^{7,8,10-13,16}$

Importantly, myeloid malignancy patients with TET2 mutations or deletions in some cases simultaneously develop lymphoid disorders: B-cell lymphoma or T-cell lymphoma. ${ }^{17}$ Among cases of human lymphoid malignancies TET2 abnormalities are the most frequently observed in T cell lymphoma (11.9\%) (especially in angioimmunoblastic $\mathrm{T}$ cell lymphoma with the frequency exceeding $30 \%$ ) and B cell lymphoma (2\%). ${ }^{3}$ The recent analysis of mutation status in the most common types of childhood leukemia revealed TET2 mutations in $3.8 \%$ of AML patients and no mutations in ALL patients. $^{18}$

Alternatively to loss-of-function mutations and deletions, promoter hypermethylation is known to be the mechanism of tumor suppressor gene inactivation. In low-grade diffuse gliomas lacking $I D H 1 / 2$ mutations, in the absence of any TET2 inactivating mutations, TET2 promoter hypermethylation was detected in $14 \%$ of analyzed patients, indicating epigenetic changes as the potential mechanism of pathogenesis in this group of glioma patients. ${ }^{19}$ Hypermethylation of the $\mathrm{CpG}$ island at the TET2 5' untranslated region was also found in $4.4 \%$ of patients with Ph-negative MPN. ${ }^{20}$ In contrast, other studies did not identify hypermethylation at TET2 promoter in MPN, MDS, CMML nor AML. ${ }^{7,15}$ Additionally TET2 promoter has been found to be methylated in MEG-01 and K562 cell lines established from blastic transformation of CML patients. ${ }^{20}$

Since the TET2 promoter methylation status in pediatric ALL has not been reported, the aim of the present study was to determine if promoter hypermethylation may be a mechanism of TET2 inactivation in a group of 45 childhood
Correspondence: Ewa Musialik, Department of Molecular and Translational Oncology, Maria Sklodowska-Curie Memorial Cancer Center and Institute of Oncology, W.K. Roentgena 5, 02-781 Warsaw, Poland.

Tel.: +48.225.462.898 - Fax: +48.225.463.191.

E-mail:musialik.ewa@gmail.com

Key words: TET2, B-cell acute lymphoblastic leukemia, DNA methylation.

Contributions: EM, conception and design of the study, acquisition, analysis and interpretation of data, drafting of manuscript, critical revisions for intellectual content; MB, acquisition, analysis and interpretation of data, drafting of manuscript, critical revisions for intellectual content; $\mathrm{AW}$, acquisition, analysis and interpretation of data; MM, drafting of manuscript, critical revisions for intellectual content; JAS, conception and design of the study, drafting of manuscript, critical revisions for intellectual content.

Conflict of interests: the authors declare no potential conflict of interests.

Funding: this work was supported by the research grant No. 344/N-INCA/2008/0 from Ministry of Science and Higher Education, Poland.

Received for publication: 31 January 2014.

Accepted for publication: 24 March 2014.

This work is licensed under a Creative Commons Attribution NonCommercial 3.0 License (CC BYNC 3.0).

(C) Copyright E. Musialik et al., 2014

Licensee PAGEPress, Italy

Hematology Reports 2014; 6:5333

doi:10.4081/hr.2014.5333

ALL cases. We also evaluated the level of TET2 expression in pediatric ALL.

\section{Materials and Methods}

\section{Patients}

Forty-five pediatric acute lymphoblastic leukemia patients were included. Clinical features of patients are summarized in Table 1 and Supplementary Table 1. The study was approved by the local ethical committee. Thirty-eight samples were diagnosed as ALL Bcommon, 2 as ALL-proB, 3 ALL B-common/preB and 2 as ALL-mature B. Ten peripheral blood mononuclear cells (PBMC) samples obtained from anonymous pediatric donors without evidence of any hematological disorders served as a control. Additional six normal PBMC samples were used for the isolation of CD19-positive cells with CD19 MicroBeads Kit and MACS separation columns (Miltenyi Biotec, Auburn, CA, USA). The purity of CD19+ enriched fraction 
was verified by flow cytometry (FACSCanto II, BD Bioscience, San Jose, CA, USA) and ranged between 71.0-98.1\% (details in Supplementary Figure 1).

\section{TET2 promoter methylation analysis}

DNA was extracted from 29 ALL PBMC samples, 16 whole blood ALL samples and 10 control PBMC samples using QIAamp DNA Mini Kit (Qiagen, Hilden, Germany). DNA quantity was measured using NanoDrop (Thermo Scientific, Wilmington, DE, USA). DNA was bisulfite converted using EpiTect kit (Qiagen), according to manufacturer's recommendations. Methylation-specific PCR (MSP) was carried out using TET2 promoter methylation and non-methylation specific primer pairs reported previously. ${ }^{20}$ The PCR was carried out in $15 \mu \mathrm{L}$ containing $10 \times$ PCR buffer with $\mathrm{MgCl}_{2}, 0.25 \mathrm{mM}$ dNTPs, 3 pmol of each primer, 0.6 U FastStart Taq DNA polymerase (Roche Applied Science, Mannheim, Germany). PCR conditions were as follows: initial denaturation for $3 \mathrm{~min}$ at $94^{\circ} \mathrm{C}$, followed by 40 cycles of $30 \mathrm{~s}$ at $94^{\circ} \mathrm{C}, 30 \mathrm{~s}$ at $64^{\circ} \mathrm{C}$ temperature, $30 \mathrm{~s}$ at $72^{\circ} \mathrm{C}$, and final elongation $7 \mathrm{~min}$ at $72^{\circ} \mathrm{C}$. DNA from normal human PBMC was methylated in vitro with SssI DNA methyltransferase (New England Biolabs, Beverly, MA, USA), according to manufacturer's recommendation and used as a positive control. Commercially available universal unmethylated human DNA served as a negative control (Qiagen). The amplified products were electrophoresed in $8 \%$ polyacrylamide gel and stained with ethidium bromide. The presence of methylation specific PCR product was classified as a positive result. To verify positive result, bisulfite sequencing has been applied. Methylation positive PCR products from ALL patient number 3 and positive control were cloned into plasmid vector using StrataClone cloning kit (Agilent Technologies, Mississauga, ON, Canada). Individual bacterial colonies were subjected to sequencing using the BigDye Terminator v3.1 chemistry (Applied Biosystems, Foster City, CA, USA) and 3130xl Genetic Analyzer (Applied Biosystems). Sequencing results were analyzed and presented with the use of $\mathrm{BiQ}$ Analyzer.

\section{TET2 expression level analysis}

The TET2 expression was examined in 27 ALL patients, 10 pediatric PBMC and 6 pediatric CD19+ control samples. Total RNA was isolated from PBMC samples using RNAqueous-Micro Kit (Ambion, Austin, TX, USA) according to manufacturer's protocol. RNA quantity was measured using NanoDrop (Thermo Scientific). 500 ng of each RNA sample was reverse-transcribed using SuperScript
II Reverse Transcriptase (Invitrogen, Paisley, UK). The real-time reverse transcription polymerase chain reaction was performed in 7900HT Fast Real-Time PCR System (Applied Biosystems). The PCR reaction was carried in $5 \mu \mathrm{L}\left(1.5 \mu \mathrm{L} 10 \times\right.$ diluted cDNA template, $\mathrm{RT}^{2}$ SYBR Green qPCR Mastermix (Qiagen) and $0.25 \mu \mathrm{M}$ of forward and reverse primer). PCR conditions were as follows: $95^{\circ} \mathrm{C}$ for $10 \mathrm{~min}, 45$ cycles: $95^{\circ} \mathrm{C}$ for $15 \mathrm{~s}$ and $60^{\circ} \mathrm{C}$ for $60 \mathrm{~s}$. Ubiquitin $(U B C)$ gene was used as a reference (details in Supplementary Figure 2). The primer pairs sequences were: TET2 forward: 5' AAGGAGACCCGACTGCAACTG 3', TET2 reverse: 5' TCTTGAGAGGGTGTGCTGCTG 3', $U B C$ forward: 5' ATTTGGGTCGCGGTTCTTG 3', $U B C$ reverse: 5' TGCCTTGACATTCTCGATGGT 3'. TET2 expression was calculated by the $2^{-\Delta \mathrm{Ct}}$ method, where $\mathrm{Ct}$ was defined as a difference between Ct value for TET2 and $U B C$.

\section{Statistical analysis}

The difference in TET2 expression level between ALL patients and control samples was evaluated by two sided Mann-Whitney test with a significance threshold level $\alpha=0.05$. Calculations were performed using GraphPad Prism software (La Jolla, CA, USA).

\section{Results}

Methylation specific PCR analysis of acute lymphoblastic leukemia samples

The methylation-specific PCR analysis was successfully performed for all the samples. TET2 promoter methylation was detected in one of 45 analyzed pediatric acute lymphoid leukemias - patient number 3 (Figure 1A). The patient was 14-year old girl diagnosed with ALL B-common without central nervous system involvement (with additional copy of $A B L$ gene). The methylated MSP product was visible as a faint band on the polyacrylamide gel. All the other ALL as well as pediatric control blood samples were methylation negative.

The methylation of TET2 promoter in positive patient and positive control DNA were further assessed by cloning of methylation specific PCR reaction product and sequencing of plasmid DNA from individual bacterial colonies. Analysis of eight clones revealed the heterogeneous methylation pattern with methylation of $45 \%$ of CpGs within amplicon from patient's 3 sample and near complete DNA methylation in control DNA (Figure 1B and C).

Table 1. Acute lymphoblastic leukemia patients characteristic.

\begin{tabular}{lc}
\hline Patients characteristics & Number of patients (\%) \\
Age at diagnosis (mean: $6.3 ;$ range: 1-17) & \\
1-9 years & $32 / 43(74)$ \\
$10-18$ years & $11 / 43(26)$ \\
Sex & \\
Male & $21 / 45(47)$ \\
Female & $24 / 45(53)$ \\
\hline Leukocyte count at diagnosis, $\times 10^{3} / \mathrm{L}$ (mean: 24; range 1.4-181) & \\
$<50$ & $37 / 43(86)$ \\
$>50$ & $6 / 43(14)$ \\
Central nervous system involvement & \\
Yes & $3 / 43(7)$ \\
No & $40 / 43(93)$ \\
\hline Cell lineage & \\
Pro-B ALL & $2 / 45(4)$ \\
Common precursor B-cell ALL & $38 / 45(84)$ \\
Pre-B ALL & $3 / 45(7)$ \\
Pre-B ALL & $2 / 45(4)$ \\
Chromosome number & \\
Normal & $18 / 42(43)$ \\
$<45$ chromosomes & $1 / 42(2)$ \\
45 chromosomes & $0 / 42(0)$ \\
$46-51$ chromosomes & $2 / 42(5)$ \\
$>51$ chromosomes (hyperdiploidy) & $14 / 42(33)$ \\
Other* & $7 / 42(17)$ \\
Chromosomal translocations & \\
Philadelphia chromosome (BCR-ABL, t $(9 ; 22))$ & $0 / 43(0)$ \\
MLL (1lq23) translocations & $1 / 43(2)$ \\
\hline & \\
\hline
\end{tabular}

ALL, acute lymphoblastic leukemia. *Other: near tetraploidy (>80 chromosomes) (1 patient), 46 chromosomes with the presence of deletion(s), addition(s) and/or translocations (5 patients), complex karyotype (1 patient). 


\section{TET2 expression analysis}

TET2 expression levels of B-cell ALL patients and control samples from pediatric healthy donors were compared. The mean TET2 expression level in ALL patients was lower in comparison to pediatric PBMC and CD19+ control samples with the fold change 2.45 and 4.52 , respectively $(\mathrm{P}<0.0001$ and $\mathrm{P}=0.0002$, respectively) (Figure 1D). The lowest expression level was detected in a methylation positive sample (patient number 3). TET2 expression level was higher in CD19+ cells isolated from healthy donors in comparison to PBMC control samples with the fold change $1.84(\mathrm{P}=0.0047)$.

TET2 expression level was not correlated with white blood cells count nor patients' age. As the lower TET2 expression level in immature hematopoietic cells in comparison to differentiated B-cells was observed, ${ }^{6}$ gene expression level was additionally analyzed in the context of blasts percentage in ALL samples. No significant correlation between TET2 expres- sion level and percentage of blasts treated as continuous variable was observed, however some trend was found when patients were grouped into four categories according to blasts number (Figure 1E). A tendency of lower TET2 expression in samples with higher blasts number was observed with a significant difference between patients with $1-25 \%$ and $51-75 \%$ blast cells $(\mathrm{P}=0.0127)$. Interestingly, 5 samples with blasts number over $75 \%$ did not match this trend and revealed high gene expression.

methDNA unmethDNA patient1 patient2 patient3

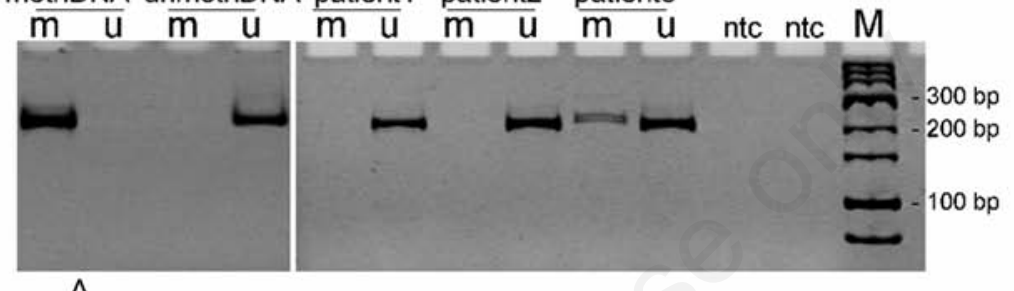

A

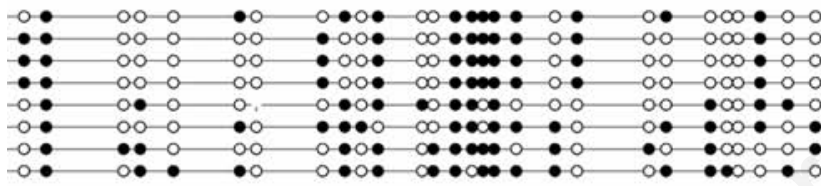

B

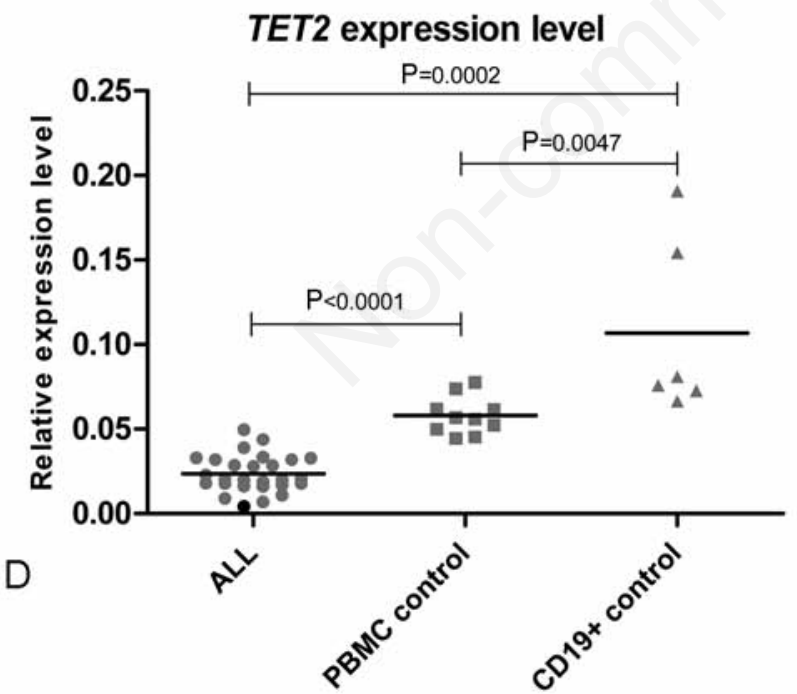

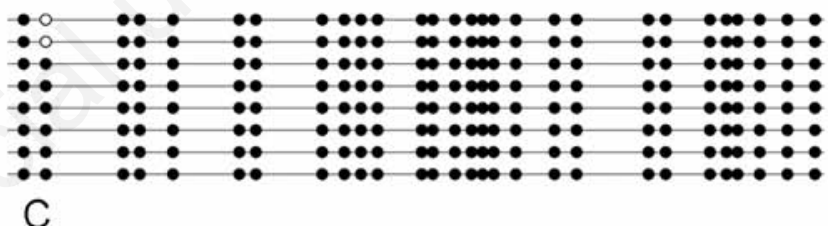

TET2 expression level

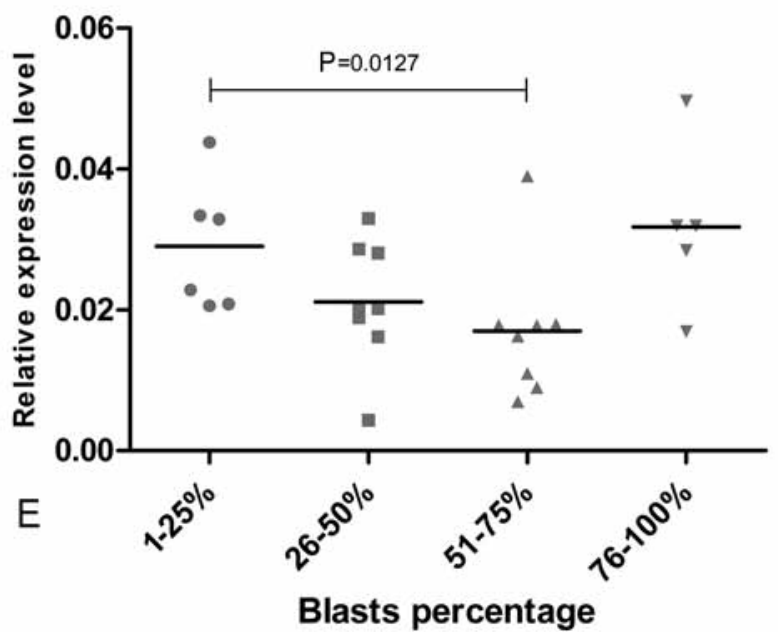

Figure 1. (A) An example of methylation-specific PCR result of TET2 promoter methylation analysis. (1-3) Acute lymphoblastic leukemia (ALL) patients, patient number 3 is the one with TET2 promoter methylation: $\mathrm{M}$ - product amplified with primers specific for methylated TET2 sequence, $\mathrm{U}$ - product amplified with primers specific for unmethylated TET2 sequence; meth - methylated control DNA (normal human DNA methylated in vitro with SssI DNA methyltransferase), unmeth - unmethylated control DNA (commercially available universal unmethylated human DNA); NTC - no template control; M - marker. (B-C) DNA sequencing analysis of methylation specific PCR product in ALL patient number 3 (B) and methylation positive control (C). Each sequenced clone is represented by a row. Black circles correspond to methylated Cs, white circles correspond to unmethylated Cs, and small vertical lines without a circle correspond to missing values (e.g. caused by sequencing errors). (D) The comparison of TET2 expression level in ALL patients, normal peripheral blood mononuclear cells (PBMCs) and isolated normal CD19+ cells. Patient number 3 (TET2 methylation positive) is marked with a black dot. (E) The comparison of TET2 expression levels between ALL patients stratified according to blasts percentage. 


\section{Discussion}

TET2 inactivating mutations and deletions frequently occur in myeloid disorders including MPD, MDS, CMML and AML. Two AML patients with TET2 locus deletions who simultaneously developed malignant lymphoma were reported. ${ }^{3}$ Frameshifts and nonsense TET2 mutations in groups of B and T lymphoma patients have been identified with the frequency $2.0 \%$ and $11.9 \%$ respectively. One of these patients showed simultaneous deletion including TET2 locus. Additionally, experiments on two different murine models demonstrate that TET2 deficiency affects the development of cells of both myeloid and lymphoid lineages. ${ }^{3}$ Therefore TET2 appears to be potentially involved not solely in pathogenesis of myeloid but also lymphoid malignancies.

Since no TET2 mutations were found in pediatric ALL in this study we focused on the possible involvement of gene promoter hypermethylation in development of childhood B-cell ALL. ${ }^{18}$ Aberrant TET2 methylation was previously found in MPN and glioma patients. ${ }^{19,20}$

We assessed the TET2 promoter methylation status in a group of 45 pediatric ALL patients by MSP test. Methylation of TET2 promoter region in one ALL B-common patient was observed using MSP and subsequently in DNA sequencing of methylation positive PCR product. This revealed heterogeneous methylation pattern in which $45 \%$ CpG sites were methylated (Figure 1B). Promoter methylation has not been found in any of normal childhood blood samples involved.

This result shows that some degree of DNA methylation in TET2 promoter region may occur in pediatric B-cell ALL with a low incidence. Nevertheless the low frequency of TET2 methylation and reported previously lack of somatic mutations may indicate the limited role of this gene in pediatric B-cell ALL pathogenesis in contrary to myeloid neoplasms.

TET2 expression analysis revealed decreased TET2 mRNA level in ALL patients in comparison to normal children's blood samples and isolated CD19+ B-cells. The only sample positive in TET2 methylation screening revealed the lowest gene expression level. However, due to the low methylation frequency and the low scatter of expression results among leukemia patients we cannot assume that promoter methylation downregulates TET2 expression in B-cell ALL. The difference between leukemia and normal samples may result from other reasons. In our study TET2 expression was higher in isolated normal Bcells compared to normal PBMC samples from children which is in line with previously reported results. In adults TET2 was proven to be variably expressed among different blood cells populations with the highest mRNA level in granulocytes and B-lymphocytes whereas low gene expression was observed in hematopoietic progenitor cells. ${ }^{6}$ This may suggest that lower TET2 expression in leukemia patients is a result of presence of immature leukemic blasts. This is supported by the observation that TET2 level tends to decrease in leukemia samples with higher blast cells proportion. Similarly, in MDS patients characterized by disturbed normal granulocytic differentiation diminished TET2 level was observed, irrespective of the mutation status. ${ }^{6}$

As the gene expression decrease can be explained by the presence of abnormally differentiated B-cells in ALL blood samples, the difference in expression level between leukemia and normal samples should not be directly interpreted as an involvement of TET2 in leukemogenesis. Generally, TET2 promoter hypermethylation seems to have no clinical relevance in childhood B-cell ALL due to its low frequency. This study did not show the relationship between promoter methylation and gene expression and we observed stable expression level in B-cell ALL samples with the low scatter of qRT-PCR results. It cannot be ruled out that TET2 promoter methylation may play role in some rare ALL patients, but it's evaluation would require a large number of samples and does not seem to be reasonable.

\section{Conclusions}

TET2 promoter hypermethylation is a rare event in childhood B-cell ALL therefore it does not seem to have a clinical relevance in this group of leukemia patients.

\section{References}

1. Ito S, D'Alessio AC, Taranova OV, et al. Role of Tet proteins in $5 \mathrm{mC}$ to $5 \mathrm{hmC}$ conversion, EScell self-renewal and inner cell mass specification. Nature 2010;466:1129-33.

2. Ko M, Huang Y, Jankowska AM, et al. Impaired hydroxylation of 5-methylcytosine in myeloid cancers with mutant TET2. Nature 2010;468:839-43.

3. Quivoron C, Couronné L, Della Valle V, et al. TET2 inactivation results in pleiotropic hematopoietic abnormalities in mouse and is a recurrent event during human lymphomagenesis. Cancer Cell 2011;20:25-38.

4. Delhommeau F, Dupont S, Della Valle V, et al. Mutation in TET2 in myeloid cancers. N Engl J Med 2009;360:2289-301.

5. Ballestar E, Pérez C, Martínez-Calle N, et al. TET2 mutations are associated with specific 5-methylcytosine and 5-hydroxymethylcytosine profiles in patients with chronic myelomonocytic leukemia. PLoS One 2012;7: e31605.

6. Langemeijer SMC, Kuiper RP, Berends M, et al. Acquired mutations in TET2 are common in myelodysplastic syndromes. Nat Genet 2009;41:838-42.

7. Abdel-Wahab 0, Mullally A, Hedvat C, et al. Genetic characterization of TET1, TET2, and TET3 alterations in myeloid malignancies. Blood 2009;114:144-7.

8. Kosmider 0, Gelsi-Boyer V, Ciudad M, et al. TET2 gene mutation is a frequent and adverse event in chronic myelomonocytic leukemia. Haematologica 2009;94:1676-81.

9. Tefferi A. Novel mutations and their functional and clinical relevance in myeloproliferative neoplasms: JAK2, MPL, TET2, ASXL1, CBL, IDH and IKZF1. Leukemia 2010;24: 1128-38.

10. Kosmider 0, Gelsi-Boyer V, Cheok M, et al. TET2 mutation is an independent favorable prognostic factor in myelodysplastic syndromes (MDSs). Blood 2009;114:3285-91.

11. Nibourel 0, Kosmider 0, Cheok M, et al. Incidence and prognostic value of TET2 alterations in de novo acute myeloid leukemia achieving complete remission. Blood 2010;116:1132-5.

12. Metzeler KH, Maharry K, Radmacher MD, et al. TET2 mutations improve the new European LeukemiaNet risk classification of acute myeloid leukemia: a Cancer and Leukemia Group B study. J Clin Oncol 2011;29:1373-81.

13. Chou W-C, Chou S-C, Liu C-Y, et al. TET2 mutation is an unfavorable prognostic factor in acute myeloid leukemia patients with intermediate-risk cytogenetics. Blood 2011;118:3803-10.

14. Figueroa ME, Abdel-Wahab O, Lu C, et al. Leukemic IDH1 and IDH2 mutations result in a hypermethylation phenotype, disrupt TET2 function, and impair hematopoietic differentiation. Cancer Cell 2010;18:553-67.

15. Jankowska AM, Szpurka H, Tiu R V, et al. Loss of heterozygosity 4q24 and TET2 mutations associated with myelodysplastic/myeloproliferative neoplasms. Blood 2009;113:6403-10.

16. Tefferi A, Pardanani A, Lim K-H, et al. TET2 mutations and their clinical correlates in polycythemia vera, essential thrombocythemia and myelofibrosis. Leukemia 2009;23:905-11.

17. Viguié F, Aboura A, Bouscary D, et al. Common 4q24 deletion in four cases of hematopoietic malignancy: early stem cell involvement? Leukemia 2005;19:1411-5.

18. Langemeijer SMC, Jansen JH, Hooijer J, et al. TET2 mutations in childhood leukemia. Leukemia 2011;25:189-92.

19. Kim Y-H, Pierscianek D, Mittelbronn M, et al. TET2 promoter methylation in low-grade diffuse gliomas lacking IDH1/2 mutations. J Clin Pathol 2011;64:850-2.

20. Chim CS, Wan TS, Fung TK, Wong KF. Methylation of TET2, CBL and CEBPA in Phnegative myeloproliferative neoplasms. J Clin Pathol 2010;63:942-6. 